\title{
Survival prediction in patients with resectable colorectal liver metastases: Clinical risk scores and tumor response to chemotherapy
}

\author{
DA XU ${ }^{1 *}$, XIAO-FENG LIU ${ }^{2 *}$, XIAO-LUAN YAN ${ }^{1}$, KUN WANG $^{1}$ and BAO-CAI XING ${ }^{1}$
}

\begin{abstract}
${ }^{1}$ Hepatopancreatobiliary Surgery Department I, Key Laboratory of Carcinogenesis and Translational Research, Ministry of Education, Peking University School of Oncology, Beijing Cancer Hospital and Institute, Beijing 100142; ${ }^{2}$ Department of Cell Biology, School of Basic Medical Research, Peking University Health Science Center, Beijing 100191, P.R. China
\end{abstract}

Received April 21, 2017; Accepted October 12, 2017

DOI: $10.3892 / \mathrm{ol} .2017 .7191$

\begin{abstract}
Clinical risk scores and response to pre-operative chemotherapy are prognostic factors of colorectal liver metastases. The aim of the present study was to evaluate the effectiveness of combining these factors to predict patient survival and to select patients for curative therapy. The study included 189 patients who underwent hepatectomy following neo-adjuvant chemotherapy, for initially resectable colorectal liver metastases, between January 2005 and December 2015. Patients were stratified into four sub-groups: A1-2, low clinical risk scores with/without a response to pre-operative chemotherapy; and B1-2, high clinical risk scores with or without a response to pre-operative chemotherapy. Treatment and survival data were analysed. Survival was significantly longer in patients with low clinical risk scores and a response to pre-operative chemotherapy; these factors were confirmed as independent prognostic factors by multivariate analysis. Combining clinical risk score and chemotherapy response classification, patient survival was significantly longer for groups A1-2/B1 compared with for group B2, in which only $10.2 \%$ of patients were alive after 5 years. Of those with no response to first-line chemotherapy, survival was significantly longer in patients who responded to second-line chemotherapy.
\end{abstract}

Correspondence to: Professor Kun Wang or Professor Bao-Cai Xing, Hepatopancreatobiliary Surgery Department I, Key Laboratory of Carcinogenesis and Translational Research, Ministry of Education, Peking University School of Oncology, Beijing Cancer Hospital and Institute, 52 Fucheng Road, Haidian, Beijing 100142, P.R. China

E-mail: wang-kun@vip.sina.com

E-mail: xingbaocai88@sina.com

*Contributed equally

Key words: clinical risk score, colorectal liver metastasis, neo-adjuvant chemotherapy, prognostic factor, survival, tumor response
A combined clinical risk score and chemotherapy response classification may aid in identifying suitable candidates for potentially curative therapy.

\section{Introduction}

Colorectal cancer (CRC) constitutes the third most frequent type of cancer worldwide (1). Approximately $50 \%$ of all patients with CRC develop liver metastases (LMs) $(2,3)$. Hepatectomy is generally regarded as the most effective and potentially curative treatment for patients with colorectal liver metastases (CRLMs), with a 5-year survival rate of $40-50 \%$ (4-6). Due to recent advances in the development of surgical techniques and peri-operative therapy, an aggressive approach to hepatic metastases resection has been widely adopted. However, not all patients with technically resectable liver-limited metastases benefit from surgery, with $>50 \%$ of patients developing recurrence within 2 years after resection $(7,8)$. Many prognostic factors have emerged for predicting survival in patients with CRLMs after hepatectomy, the most common criteria for patient selection and recurrence prediction are based on the clinical risk score system (CRS) presented by Fong et al (9). Numerous studies have confirmed that patients in high-risk group $(\mathrm{CRS} \geq 3)$ would have a significantly shorter overall survival (OS) than patients in the low-risk $(\mathrm{CRS}<3)$ group (6,10-12). However, the validity of CRS has recently been debated as it was based on treatment outcomes of patients in 1990s, prior to the implementation of current chemotherapy regimens $(13,14)$.

Neo-adjuvant chemotherapy (NACT) has been widely adopted for the treatment of patients with resectable CRLMs, not only in prolonging progression-free survival (15), but also in downsizing the tumors to preserve a larger volume of liver parenchyma, making surgery easier $(16,17)$. However, tumor progression does occur in approximately $5-10 \%$ of patients after NACT $(18,19)$. Numerous studies have demonstrated that a tumor's response to pre-operative chemotherapy (TRC) is an important predictive factor for evaluating long-term survival in patients with CRLMs (18-20). However, whether patients could benefit from liver resection after tumor progression during NACT remains controversial. In addition, although 
both the Fong's CRS system (9) and the TRC can predict a patient's oncological status, it is unknown whether a combination of the CRS system and the TRC (CRS-TRC classification) could improve the predictive accuracy of survival in patients with CRLMs. Therefore, the aim of our study was to evaluate which patients are suitable for hepatectomy after underwent tumor pregression during chemotherapy, and the value of CRS-TRC classification in predicting survival and selecting patients with CRLMs for curative therapy.

\section{Materials and methods}

Patient selection. A total of 425 patients with CRLMs underwent hepatectomy between January 2005 and December 2015 in the Hepatopancreatobiliary Surgery Department I at the Beijing Cancer Hospital and Institute (Beijing, China). Patients were screened on the following exclusion criteria: Concurrent unresectable extrahepatic metastases; no R0 resection; repeated hepatectomy due to liver recurrences; and unavailable TRC data. All study participants provided informed written consent, and the study was approved by the Ethical Review Board committee of the Beijing Cancer Hospital and Institute.

Study design. The tumor response was evaluated after every two cycles of NACT based on computed tomography (CT) or magnetic resonance (MR) images, using the Response Evaluation Criteria in Solid Tumors (v.1.1) (21). The good TRC group (response to NACT) included patients with a complete or partial response and those with a response within a stable disease status (a reduction in the sum of tumor diameters of $<30 \%$ ), while the bad TRC group comprised of patients with a progressive disease or progression within a stable disease status (an increase in the sum of the diameters of the target lesion of $<20 \%$ ). In patients who received multiple lines of chemotherapy, the tumor response to the last regimen was considered. According to the CRS system patients were classified into a either a high-risk $(\mathrm{CRS} \geq 3)$ or a low-risk (CRS <3) group (9). We further subclassified patients using our new combined CRS-TRC classification, shown in Fig. 1, into the following subgroups: Group A1, low CRS and good TRC; group A2, low CRS and bad TRC; group B1, high CRS and good TRC; and group B2, high CRS and bad TRC. Clinical outcomes and survival were evaluated between these subgroups.

Pre-operative management and neo-adjuvant chemotherapy. A multidisciplinary team meeting is routinely conducted each week at our centre. Gadoxetic acid/contrast-enhanced MR imaging was routinely performed in every CRLMs patients. Positron emission tomography-CT scans performed for patients with suspected extrahepatic metastases. In general, patients received 2-6 cycles of NACT (22). A modern chemotherapy regime for all cases, using oxaliplatin- or irinotecan-based chemotherapy, in combination with targeted therapy using cetuximab or bevacizumab determined on a per-patient basis using the $R A S$ mutation status. The time interval between the date of the last chemotherapy session and hepatic surgery was usually 4 weeks, extending to $6-8$ weeks for patients who received bevacizumab.
Patient selection for liver resection and operative technique. LMs were considered resectable provided the following criteria were met: i) The possibility of R0 resection with a liver remnant of $\geq 30 \%$ and sufficient hepatic blood inflow and outflow, and ii) no evidence of unresectable extrahepatic metastases $(23,24)$. Hepatic resections were performed using the parenchymal sparing method (9) with a resection margin of $>1 \mathrm{~mm}$. An ablation technique $(25,26)$ was performed, in combination with resection surgery, for tumors that were deep and technically difficult to resect.

Post-operative outcomes and follow-up. Contrast-enhanced CT scans or MR imaging, liver function and carcinoembryonic antigen levels were performed every 3 months within the first 2 years, and then every 6 months after operation.

Statistical analysis. Continuous variables were presented as the mean and standard deviation or the median and interquartile range. Discreet variables were presented as the number and percentage. Between-group differences were evaluated using a chi-squared test for categorical variables, and Student's t-test or non-parametric test for continuous variables, as appropriate. Disease-free survival (DFS) and OS were calculated from the date of hepatectomy. Patients were followed until death or the end-point of the study (April 1, 2016), whichever occurred first. Survival curves were plotted using the Kaplan-Meier method and compared using the log-rank test. Variables that were statistically significant in the univariate analysis $(\mathrm{P}<0.10)$ were included in the multivariate analysis using a Cox proportional hazards model. All statistical analyses were conducted using Statistical Package for the Social Sciences for Windows v.21.0 (IBM SPSS, Armonk, NY, USA). P<0.05 was considered to indicate a statistically significant difference.

\section{Results}

A total of 195 patients with resectable CRLMs underwent pre-operative chemotherapy followed by surgery. Six patients were lost to follow-up (Fig. 1). Therefore, our analysis was based on the data of 189 patients. The following data from these patients was retrieved for analysis: Age, sex, primary tumor status, LM status, disease-free interval, carcinoembryonic antigen levels, and chemotherapy regimen used.

Patient characteristics and treatment regimens. The clinical variables and treatment regimens of the 189 patients included in our analysis are summarised in Table I. In summary, our group include 121 males and 68 females, with a mean age was 56 years. In 82 patients (43.4\%), the primary tumor was located in the rectum. Fifty-seven patients $(30.2 \%)$ were treated with targeted therapy and 36 patients $(19.0 \%)$ received multiple lines of chemotherapy. When categorised according to the proposed CRS-TRC classification, 68 patients with a low CRS (group A1) responded to chemotherapy, whereas 26 patients also with a low CRS (group A2) did not. Variables between patients in groups A1 and A2 were comparable, with the exception of the proportion who received adjuvant chemotherapy $(\mathrm{P}=0.011)$ and the use of multiple lines of chemotherapy $(\mathrm{P}<0.01)$. Sixty-one patients with a high CRS (Group B1) responded to chemotherapy, whereas 33 patients with a high CRS (Group 


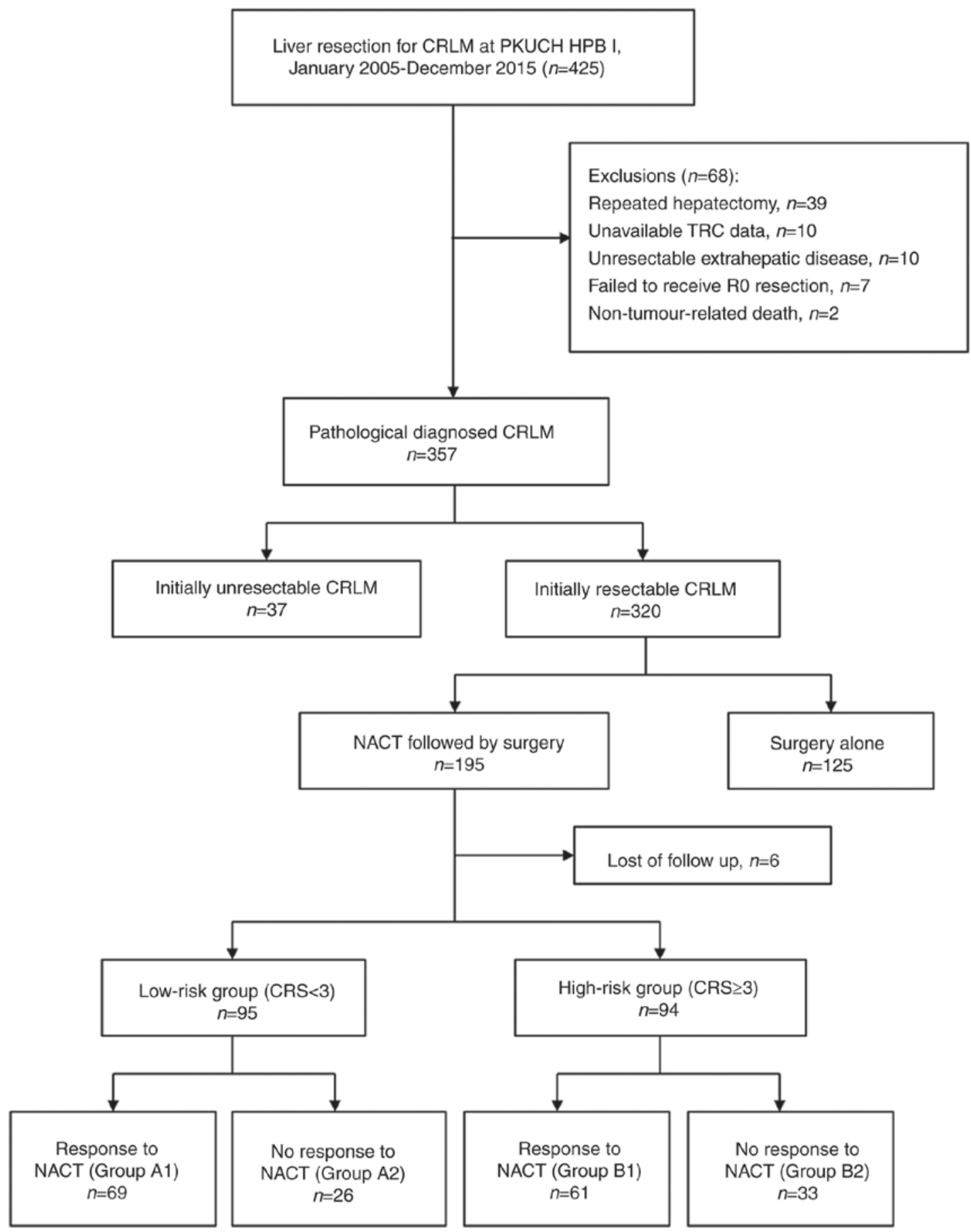

Figure 1. Study flowchart. CRLM, colorectal liver metastasis; CRS, clinical risk score; HPB I, Hepatopancreatobiliary Surgery Department I; NACT, neo-adjuvant chemotherapy; PKUCH, Peking University School of Oncology, Beijing Cancer Hospital.

B2) did not. Multiple lines of chemotherapy $(\mathrm{P}<0.001)$ were the only variable that was statistically significant between patients in Group B1 and Group B2. Chemotherapy regimens were comparable between the two sub-groups in both the lowand the high-risk groups.

Survival analysis. The median follow-up duration was 46 (95\% confidence interval [CI]: 37.7-54.3) months. The Kaplan-Meier survival curve is shown in Fig. 2. Sixty-seven patients (35.4\%) were still alive after follow-up. The median OS time was 46 (95\% CI: 37.7-54.3) months. When categorised according to the CRS system, the 1-, 3-, and 5-year OS rates were 93.0,
78.7, and $62.3 \%$ for patients in the low-risk group (CRS <3) and $90.5,36.2$, and $25.5 \%$ for patients in the high-risk group $(C R S \geq 3)$, respectively $(\mathrm{P}<0.001 ;$ Fig. $2 \mathrm{~A})$. When categorised according to TRC, the 1-, 3-, and 5-year OS rates were 95.6, 70.4, and $56.4 \%$ for patients in the TRC group and 91.2, 33.7, and $20.2 \%$ for patients in the no TRC group, respectively (P<0.001; Fig. 2B). Multivariate analysis identified both CRS and TRC as independent prognostic factors for OS in patients with CRLMs (Table II).

We subsequently categorised patients into four sub-groups according to the proposed CRS-TRC classification outlined in Fig. 1. Patients in Group A1-2 had a low CRS with/without a 


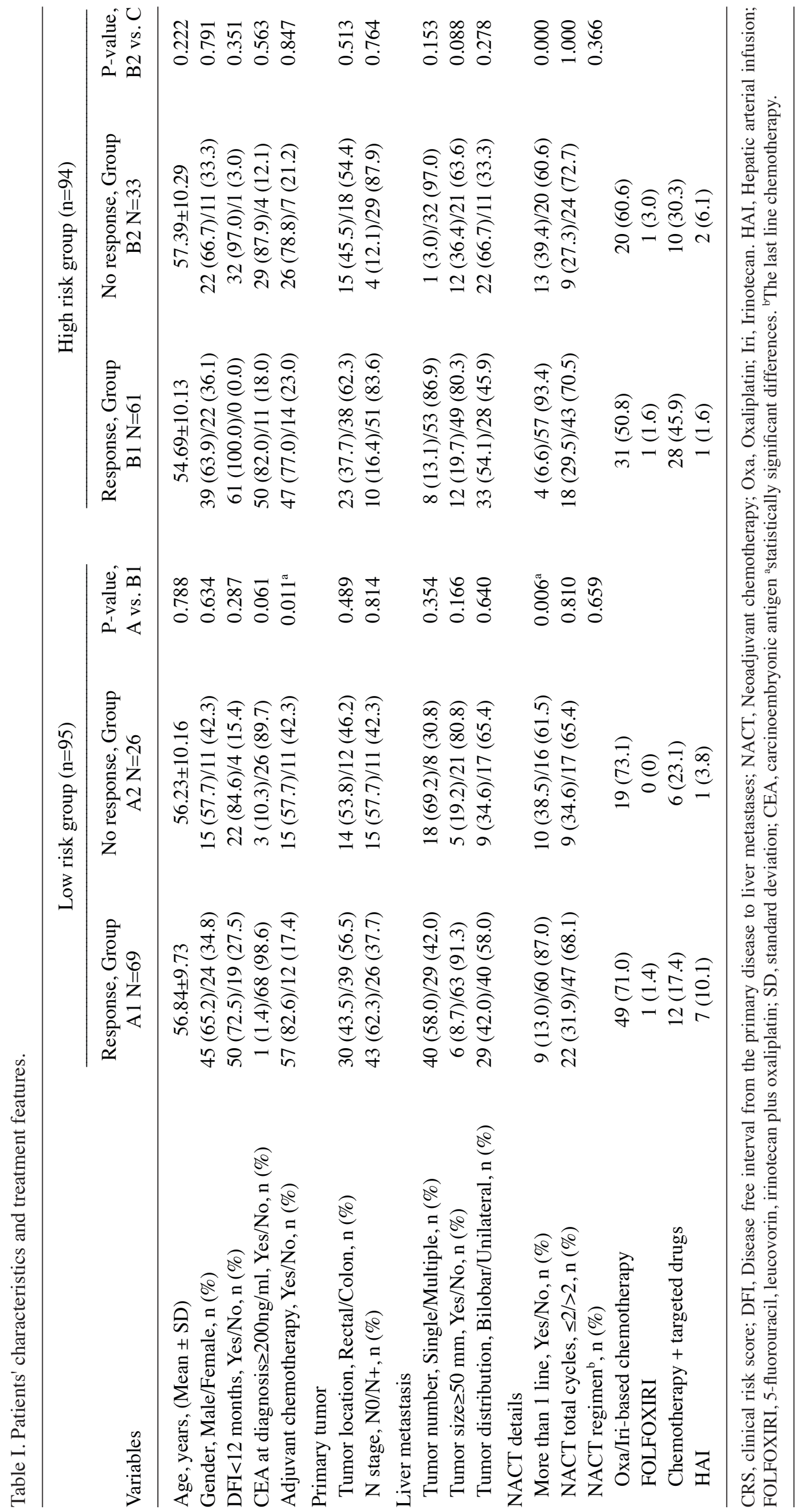


A

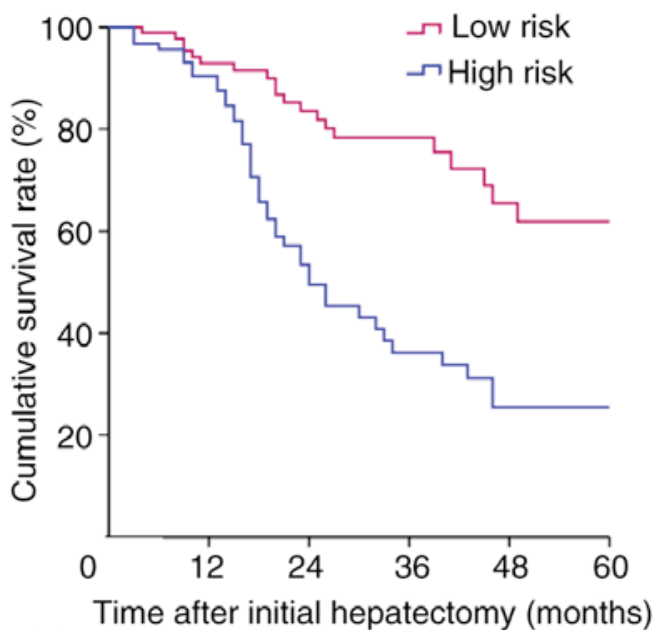

No. at risk

$\begin{array}{lllllll}\text { Low risk } & 95 & 88 & 79 & 74 & 62 & 59 \\ \text { High risk } & 94 & 85 & 46 & 34 & 24 & 24\end{array}$

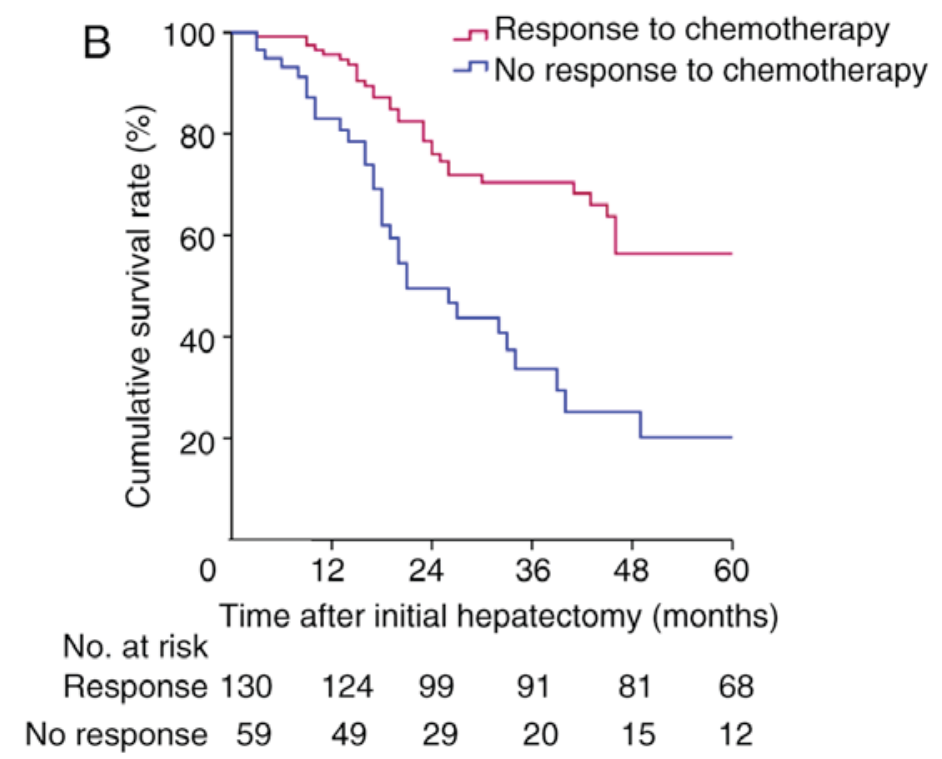

Figure 2. Cumulative survival in (A) patients with a high and low clinical risk score ( $\mathrm{P}<0.001$; log-rank test) and (B) patients with and without a response to pre-operative chemotherapy $(\mathrm{P}<0.001 ;$ log-rank test).

TRC and patients in Group B1-2 had a high CRS with/without a TRC. The Kaplan-Meier survival curve of the patients is shown in Fig. 3. The 1-, 3-, and 5-year OS rates were 96.7, 85.8, and $72.9 \%$, respectively, for patients in Group A1; 82.6, 59.5, and $31.7 \%$, respectively, for patients in Group A2, 94.3, 49.4, and $35.3 \%$, respectively, for patients in Group B1, and 83.3, 15.3, and $10.2 \%$, respectively, for patients in Group B2 $(\mathrm{P}<0.001$; Fig. 3A). The median DFS was 24.0 (95\% CI: 20.8-27.9) months, 11.0 (95\% CI: 7.8-14.2) months, 8.0 (95\% CI: 5.5-10.5) months, and 4.0 (95\% CI: 2.4-5.6) months for patients in Group A1-2 and Group B1-2, respectively ( $\mathrm{P}<0.001$; Fig. 3B).

Survival analysis of patients who progressed on first-line chemotherapy. Among the 71 patients underwent tumor progression during first-line chemotherapy, 36 were treated surgically immediately after first-line progression. The remaining 35 patients received second-line chemotherapy, with only 13 patients (37.1\%) showing a good tumor response, a proportion that is considerably lower than the 130 patients $(68.8 \%)$ who had a tumor response to first-line chemotherapy. The Kaplan-Meier survival curve of these patients is shown in Fig. 4. Patients who responded well to second-line chemotherapy had a significantly better prognosis than patients who progressed on either firstor second-line chemotherapy $(\mathrm{P}<0.05)$. Multivariate analysis identified TRC to second-line chemotherapy as an independent prognostic factor for OS in patients who did not respond to first-line chemotherapy (Table II).

To further investigate the effects of second-line chemotherapy on TRC, the treatment details of these patients were analysed (Table III). Of the 13 patients with a tumor response to second-line chemotherapy followed by surgery, six patients (46.2\%) received targeted therapy, four patients $(30.8 \%)$ underwent HAI therapy, and only three patients $(23.0 \%)$ were still treated with a doublet regimen. Conversely, of the remaining 22 patients who progressed on second-line chemotherapy, 11 patients $(50.0 \%)$ were still treated with a doublet regimen, a ratio that is considerably higher than for the TRC group.

\section{Discussion}

With respect to tumor oncological status, the CRS system of Fong has been validated by numerous studies for its efficiency in predicting survival (6,10-12). Meanwhile, with recent advances in the development of more effective chemotherapeutic and targeted agents, some authors questioned the impact of Fong's CRS system on survival, arguing that the real value of the tumor response in predicting survival may be underestimated in the modern era of chemotherapy $(13,14)$. Allen et al $(19)$ initially found that patients with synchronous CRLMs who responded well to chemotherapy had a prolonged survival compared to patients who underwent surgery alone. Similarly, Adam et al (27) reported that survival times were significantly shorter in patients with multiple metastases ( $\geq 4$ tumors) who experienced tumor progression during NACT after hepatectomy. However, whether surgery should be performed in patients who have experienced progression during NACT has long been debated $(28,29)$. Neumann et al $(28)$ reported that there was no association between TRC and long-term survival in patients with synchronous CRLMs, indicating that liver resection may not be contraindicated in these patients. It should be noted that patients' clinical risk factors differed substantially between the above-mentioned studies. Moreover, the choice of chemotherapy regimen can play a pivotal role in tumor response, with a high proportion of patients in the above-mentioned studies treated with 5-fluorouracil monotherapy.

To investigate the outcome of patients who underwent hepatectomy under different clinical and chemotherapy response conditions, we proposed a CRS-TRC classification in which patients were stratified into four sub-groups according to both clinical risk factors and tumor response factors. Our findings demonstrate that patients with a low CRS and a TRC (Group A1) derived the most benefits of hepatectomy, with a 5-year OS rate of $72.9 \%$. Conversely, patients with a high CRS and no TRC (Group B2) exhibited the poorest prognosis, with only $10.2 \%$ of patients alive after 5 years. The long-term 
Table II. Univariate and multivariate Cox proportional hazards regression analyses of factors associated with overall survival.

\begin{tabular}{|c|c|c|c|}
\hline \multirow[b]{2}{*}{ Whole group $(\mathrm{N}=189)$} & \multirow{2}{*}{$\begin{array}{c}\text { Univariate } \\
\text { P-value }\end{array}$} & \multicolumn{2}{|c|}{ Multivariate analysis } \\
\hline & & HR $(95 \%$ CI $)$ & P-value \\
\hline Primary tumor location (rectal/colon) & 0.910 & & \\
\hline Primary N stage (N0/N+) & 0.215 & & \\
\hline Tumor number (single/multiple) & $0.043^{\mathrm{a}}$ & $0.867(0.419-1.792)$ & 0.699 \\
\hline Tumor size $(<50 \mathrm{~mm} / \geq 50 \mathrm{~mm})$ & $0.005^{\mathrm{a}}$ & $1.429(0.777-2.629)$ & 0.250 \\
\hline Tumor distribution (unilateral/bilobar) & 0.185 & & \\
\hline DFI ( $<12$ months $/ \geq 12$ months $)$ & 0.230 & & \\
\hline CEA at diagnosis $(<200 \mathrm{ng} / \mathrm{ml} / \geq 200 \mathrm{ng} / \mathrm{ml})$ & $0.035^{\mathrm{a}}$ & $1.405(0.641-3.078)$ & 0.39 \\
\hline CRS (low risk/high risk) & $0.000^{\mathrm{a}}$ & $3.000(1.501-5.995)$ & $0.002^{\mathrm{a}}$ \\
\hline Tumor response ${ }^{\mathrm{b}}$ (no/yes) & $0.000^{\mathrm{a}}$ & $2.522(1.521-4.181)$ & $0.000^{\mathrm{a}}$ \\
\hline More than 1 line NACT (no/yes) & 0.208 & & \\
\hline \multicolumn{4}{|c|}{ No-response to 1st-line chemotherapy group $(\mathrm{N}=71)$} \\
\hline CRS (low risk/high risk) & $0.006^{\mathrm{a}}$ & $2.259(1.257-4.747)$ & $0.032^{\mathrm{a}}$ \\
\hline Tumor response in 2nd line (no/yes) & $0.012^{\mathrm{a}}$ & $1.837(1.184-3.265)$ & $0.045^{\mathrm{a}}$ \\
\hline
\end{tabular}

CI, confidence interval; HR, hazard ratio; DFI, disease free interval from the primary disease to liver metastases; CRS, clinical risk score; NACT, neoadjuvant chemotherapy; CI, confidence interval; HR, hazard ratio; CEA, carcinoembryonic antigen. ${ }^{a}$ statistically significant differences. ${ }^{\mathrm{b}}$ The last line chemotherapy.
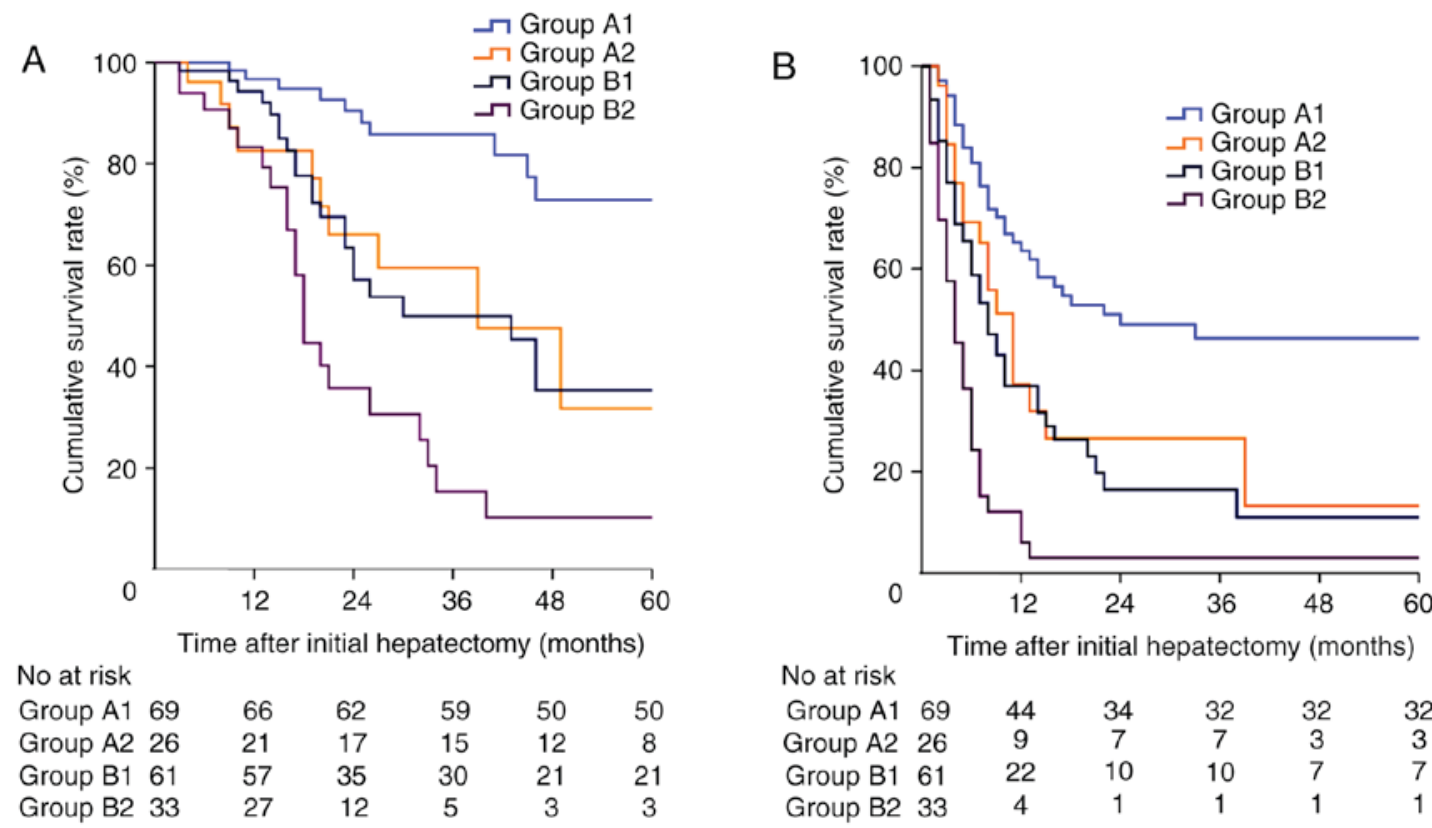

No at risk

$\begin{array}{ccccccc}\text { Group A1 } & 69 & 44 & 34 & 32 & 32 & 32 \\ \text { Group A2 } & 26 & 9 & 7 & 7 & 3 & 3 \\ \text { Group B1 } & 61 & 22 & 10 & 10 & 7 & 7 \\ \text { Group B2 } & 33 & 4 & 1 & 1 & 1 & 1\end{array}$

Figure 3. (A) Overall survival and (B) disease-free survival in patients from Group A1, Group A2, Group B1, and Group B2 (P<0.001; log-rank test).

survival of the remaining patients with only one risk factor in either the high CRS (A2) or no TRC (B1) groups was poorer than the survival among patients in Group A1, but was significantly longer than survival among patients in Group B2. These findings suggest that patients with CRLMs who have a low CRS but underwent tumor progression during NACT should not be precluded from surgical resection since the 5-year OS rate can be as high as $31.7 \%$ after hepatectomy, although no TRC is a poor prognostic factor. On the other hand, the prognosis of patients in Group B2 with both a high CRS and progressed during NACT was very poor, these patients might not be suitable for surgery, although they were initially deemed to have resectable tumors. Similarly, Vigano et al (20) found that hepatectomy should not be considered as an absolute contraindication to liver resection since a proportion of low-risk patients with disease progression would benefit from surgery. Our findings support this conclusion. In fact, in a multidisciplinary international consensus of the Expert Group on OncoSurgery management of Liver Metastases (30), oncological criteria for contraindication to hepatic resection in 


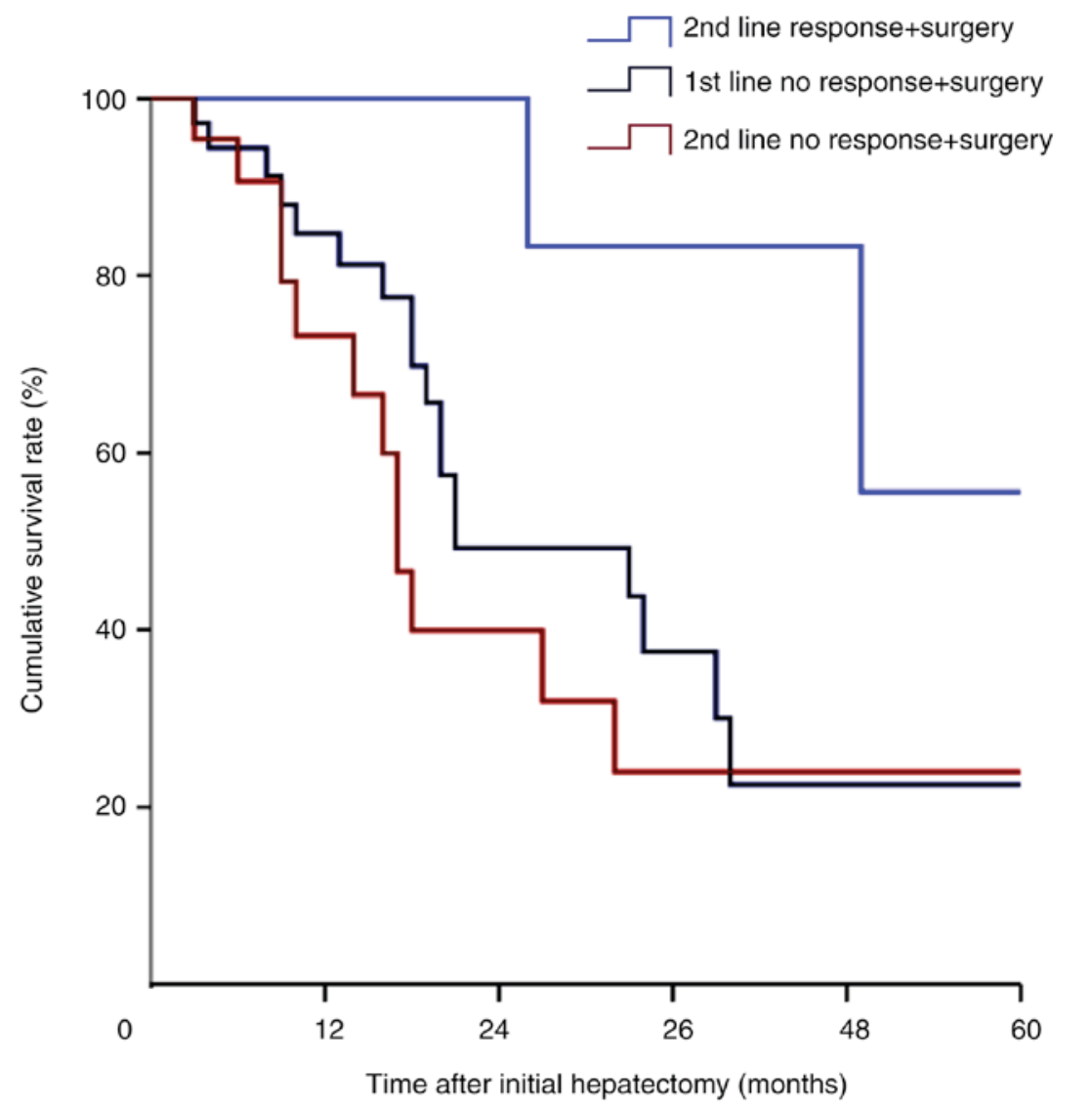

$\begin{array}{rrrrrrr}\text { No. at risk } & & & & & 11 & 7 \\ \text { 2nd line response+surgery } & 13 & 13 & 13 & 13 & 8 & 8 \\ \text { 1st line no response+surgery } & 36 & 30 & 18 & 5 & 5 & 5\end{array}$

Figure 4. Cumulative survival in patients with and without a response to second-line chemotherapy followed by surgery ( $\mathrm{P}<0.05$; $\log$-rank test).

patients with CRLMs included a greater number of tumor and tumor progression during chemotherapy. These criteria are consistent with the CRS-TRC classification, which includes 'inner' clinical risk factors, reflected by Fong's CRS system, and 'external' oncological factors, reflected by the TRC. Therefore, we can better understand that both clinical risk factors and a TRC play significant roles in patient selection for surgical resection. It may be unwise to select patients or predict survival considering only one of these factors since both are important in predicting oncological behaviour.

In the present study, the survival time of patients who underwent resection after effective second-line chemotherapy was considerably longer than that of patients who underwent resection directly or patients who still underwent resection even though second-line chemotherapy was ineffective. These findings emphasize the importance of achieving 'good' tumor control to maximise the benefit of surgical resection, especially in high-risk patients. Moreover, among patients who received second-line chemotherapy, the proportion of HAI therapy or targeted drugs used in the effective second-line chemotherapy group was considerably higher than among patients with a poor tumor response to second-line chemotherapy group. A randomised controlled trial conducted by Ye et al (31) has highlighted the efficiency of cetuximab in increasing the objective response rate. Additionally, HAI therapy has also been shown to be effective in increasing the response rate in patients who progressed on first-line systematic chemotherapy (32). Therefore, we hypothesised that the increasing use of HAI therapy and targeted drugs will assist more patients in achieving a better TRC, therefore reducing disease progression and prolonging survival. Unfortunately, although the most powerful drugs were used in our second-line chemotherapy regimens, the response rate was poor overall, with only 13 patients (37.1\%) achieving a TRC. This rate of TRC was considerably lower than the 130 patients $(68.8 \%)$ who achieved a TRC with first-line chemotherapy.

To the best of our knowledge, this is the first study to have considered both clinical and chemotherapy factors in predicting outcomes for patients with CRLMs after hepatic resection. However, there are still several limitations that need to be acknowledged in the interpretation of our results for clinical practice. Firstly, this is a retrospective analysis with a limited number of patients, although no fewer than in the majority of previous studies. Therefore, the clinical effectiveness of the proposed CRS-TRC classification will need to be verified further in prospective studies with larger cohorts. Secondly, recent studies have identified other prognostic factors to predict clinical outcomes 
Table III. Treatment details of patients who received second line chemotherapy.

\begin{tabular}{|c|c|c|c|c|c|c|c|c|}
\hline \multirow[b]{2}{*}{ Group } & \multicolumn{4}{|c|}{$\begin{array}{l}\text { Response in } 2 \text { nd line chemotherapy } \\
\text { followed by surgery (Group } 1, N=13 \text { ) }\end{array}$} & \multicolumn{4}{|c|}{$\begin{array}{l}\text { No response in } 2 \text { nd line chemotherapy } \\
\text { followed by surgery (Group } 2, N=22 \text { ) }\end{array}$} \\
\hline & Patients & CRS & Regime & Cycle & Patients & CRS & Regime & Cycle \\
\hline \multicolumn{9}{|l|}{ Targeted drugs } \\
\hline Group $1, n=6(46.2 \%)$; & $\mathrm{P} 1$ & High & FOLFIRI+BEV & 2 & $\mathrm{P} 1$ & Low & FOLFIRI+BEV & 2 \\
\hline \multirow[t]{8}{*}{ Group 2, n=9 (40.9\%) } & $\mathrm{P} 2$ & Low & FOLFIRI+BEV & 5 & $\mathrm{P} 2$ & Low & FOLFIRI+BEV & 2 \\
\hline & P3 & Low & FOLFIRI+CET & 3 & $\mathrm{P} 3$ & Low & FOLFIRI+BEV & 3 \\
\hline & $\mathrm{P} 4$ & High & FOLFOX+CET & 2 & $\mathrm{P} 4$ & High & XELIRI+BEV & 4 \\
\hline & P5 & High & XELOX+CET & 2 & P5 & High & $\mathrm{XELOX}+\mathrm{BEV}$ & 3 \\
\hline & P6 & Low & FOLFOX+CET & 3 & P6 & Low & FOLFIRI+CET & 3 \\
\hline & & & & & P7 & High & FOLFIRI+CET & 4 \\
\hline & & & & & P8 & High & FOLFIRI+CET & 4 \\
\hline & & & & & P9 & High & FOLFOX+CET & 2 \\
\hline \multicolumn{9}{|l|}{ HAI } \\
\hline Group $1, n=4(30.8 \%)$; & P7 & Low & $\mathrm{Oxa}+5-\mathrm{Fu}$ & 2 & P10 & Low & $\mathrm{Oxa}+5-\mathrm{Fu}$ & 4 \\
\hline \multirow[t]{3}{*}{ Group 2, n=2 (9.1\%) } & P8 & Low & $\mathrm{Oxa}+5-\mathrm{Fu}$ & 2 & $\mathrm{P} 11$ & High & $\mathrm{Oxa}+5-\mathrm{Fu}$ & 2 \\
\hline & P9 & High & $\mathrm{Oxa}+5-\mathrm{Fu}$ & 2 & & & & \\
\hline & $\mathrm{P} 10$ & Low & $\mathrm{Oxa}+5-\mathrm{Fu}$ & 3 & & & & \\
\hline \multicolumn{9}{|l|}{ Doublet regimes } \\
\hline Group $1, \mathrm{n}=3(23.0 \%)$; & $\mathrm{P} 11$ & High & FOLFIRI & 2 & P12 & Low & FOLFIRI & 2 \\
\hline \multirow[t]{10}{*}{ Group 2, $\mathrm{n}=11(50 \%)$} & $\mathrm{P} 12$ & Low & FOLFIRI & 3 & P13 & Low & FOLFIRI & 3 \\
\hline & P13 & High & XELOX & 4 & P14 & Low & FOLFIRI & 3 \\
\hline & & & & & P15 & High & FOLFIRI & 6 \\
\hline & & & & & P16 & High & XELIRI & 2 \\
\hline & & & & & P17 & High & XELOX & 2 \\
\hline & & & & & P18 & High & XELOX & 2 \\
\hline & & & & & P19 & Low & XELOX & 2 \\
\hline & & & & & P20 & High & XELOX & 2 \\
\hline & & & & & $\mathrm{P} 21$ & Low & FOLFOX & 2 \\
\hline & & & & & $\mathrm{P} 22$ & High & FOLFOX & 2 \\
\hline
\end{tabular}

Targeted drug, chemotherapy combined with targeted drugs, including cetuximab and bevacizumab; HAI, hepatic arterial infusion; doublet regimen, chemotherapy based on oxaliplatin or irinotecan; CRS, clinical risk score; BEV, Bevacizumab; CET, Cetuximab; Oxa, Oxaliplatin; 5-Fu, 5-fluorouracil; FOLFIRI, 5-fluorouracil, leucovorin plus irinotecan; FOLFOX, 5-fluorouracil, leucovorin plus oxaliplatin; XELOX, capecitabine plus oxaliplatin; XELIRI, capecitabine plus irinotecan.

of treatment for patients with CRLMs, including the detection of $R A S / B R A F$ mutations (33-35). However, since we only began routinely detecting $R A S / B R A F$ mutations in 2012, this factor was not considered in our study. In the future, a far more complete system may be developed, combining tumor clinical factors, gene status, and chemotherapy and pathological responses, just like the CRS system presented by Fong (9).

In conclusion, the proposed CRS-TRC classification may be beneficial in the modern chemotherapy era for selecting suitable candidates for potentially curative treatment approaches. Patients with a low CRS benefit from surgical resection even if they develop tumor progression during chemotherapy. Conversely, it may be less beneficial to perform surgery in patients with a high CRS who develop tumor progression during first-line NACT. The best approach for these patients would be to select a more powerful second-line chemotherapy regime, such as targeted drugs or HAI, to maximise tumor control.

In this 10-year retrospective cohort study, we proposed a new classification considering both Clinical Risk Scores (CRS) and tumor response to pre-operative chemotherapy (TRC), which will be assistant in predicting survival and selecting suitable patients with resectable colorectal liver metastases for curative therapy.

\section{Acknowledgements}

The present study was supported by a grant (no. 81371868) from the National Nature Science Foundation of China and Beijing Municipal Administration of Hospitals Incubating Program (code: PX2016002). 


\section{References}

1. Smith JJ and D'Angelica MI: Surgical management of hepatic metastases of colorectal cancer. Hematol Oncol Clin North Am 29: 61-84, 2015.

2. Leporrier J, Maurel J, Chiche L, Bara S, Segol P and Launoy G: A population-based study of the incidence, management and prognosis of hepatic metastases from colorectal cancer. Br J Surg 93: 465-474, 2006.

3. Rees M, Tekkis PP, Welsh FK, O'Rourke T and John TG: Evaluation of long-term survival after hepatic resection for metastatic colorectal cancer: A multifactorial model of 929 patients. Ann Surg 247: 125-135, 2008.

4. Aloia TA, Vauthey JN, Loyer EM, Ribero D, Pawlik TM, Wei SH, Curley SA, Zorzi D and Abdalla EK: Solitary colorectal liver metastasis: Resection determines outcome. Arch Surg 141: 460-467, 2006

5. Simmonds PC, Primrose JN, Colquitt JL, Garden OJ, Poston GJ and Rees M: Surgical resection of hepatic metastases from colorectal cancer: A systematic review of published studies. Br J Cancer 94: 982-999, 2006.

6. Nakai T, Ishikawa H, Tokoro $\mathrm{T}$ and Okuno K: The clinical risk score predicts the effectiveness of adjuvant chemotherapy for colorectal liver metastasis. World J Surg 39: 1527-1536, 2015.

7. Kanas GP, Taylor A, Primrose JN, Langeberg WJ, Kelsh MA, Mowat FS, Alexander DD, Choti MA and Poston G: Survival after liver resection in metastatic colorectal cancer: Review and meta-analysis of prognostic factors. Clin Epidemiol 4: 283-301, 2012.

8. Petrelli NJ: Perioperative or adjuvant therapy for resectable colorectal hepatic metastases. J Clin Oncol 26: 4862-4863, 2008

9. Fong Y, Fortner J, Sun RL, Brennan MF and Blumgart LH: Clinical score for predicting recurrence after hepatic resection for metastatic colorectal cancer: Analysis of 1001 consecutive cases. Ann Surg 230: 309-321, 1999.

10. Rahbari NN, Reissfelder C, Schulze-Bergkamen H, Jäger D, Büchler MW, Weitz J and Koch M: Adjuvant therapy after resection of colorectal liver metastases: The predictive value of the MSKCC clinical risk score in the era of modern chemotherapy. BMC Cancer 14: 174, 2014.

11. Mann CD, Metcalfe MS, Leopardi LN and Maddern GJ: The clinical risk score: Emerging as a reliable preoperative prognostic index in hepatectomy for colorectal metastases. Arch Surg 139: $1168-1172,2004$

12. Ayez N, van der Stok EP, Grunhagen DJ, Rothbarth J, van Meerten E, Eggermont AM and Verhoef C: The use of neo-adjuvant chemotherapy in patients with resectable colorectal liver metastases: Clinical risk score as possible discriminator. Eur J Surg Oncol 41: 859-867, 2015.

13. Reddy SK, Kattan MW, Yu C, Ceppa EP, de la Fuente SG, Fong Y, Clary BM and White RR: Evaluation of peri-operative chemotherapy using a prognostic nomogram for survival after resection of colorectal liver metastases. HPB (Oxford) 11: 592-599, 2009.

14. Kumar R, Dennison AR, Robertson V, Jones MJ, Neal CP and Garcea G: Clinical risk scores in the current era of neoadjuvant chemotherapy for colorectal liver metastases. ANZ J Surg: Sep 12, 2016 (Epub ahead of print).

15. Nordlinger B, Sorbye H, Glimelius B, Poston GJ, Schlag PM, Rougier P, Bechstein WO, Primrose JN, Walpole ET, Finch-Jones M, et al: Perioperative FOLFOX4 chemotherapy and surgery versus surgery alone for resectable liver metastases from colorectal cancer (EORTC 40983): Long-term results of a randomised, controlled, phase 3 trial. Lancet Oncol 14: $1208-1215,2013$

16. Baize N, Gerard B, Bleiberg H, Caroli-Bosc F, Berthier F, Legendre H, Pector JC and Hendlisz A: Long-term survival of patients downstaged by oxaliplatin and 5-fluorouracil combination followed by rescue surgery for unresectable colorectal liver metastases. Gastroenterol Clin Biol 30: 1349-1353, 2006.

17. Adam R, Delvart V, Pascal G, Valeanu A, Castaing D, Azoulay D, Giacchetti S, Paule B, Kunstlinger F, Ghémard O, et al: Rescue surgery for unresectable colorectal liver metastases downstaged by chemotherapy: A model to predict long-term survival. Ann Surg 240: 644-658, 2004.

18. Vessie EL, Liu DM, Forster B, Kos S, Baxter K, Gagnon J and Klass D: A practical guide to magnetic resonance vascular imaging: Techniques and applications. Ann Vasc Surg 28: 1052-1061, 2014
19. Allen PJ, Kemeny N, Jarnagin W, DeMatteo R, Blumgart L and Fong Y: Importance of response to neoadjuvant chemotherapy in patients undergoing resection of synchronous colorectal liver metastases. J Gastrointest Surg 7: 109-117, 2003.

20. Vigano L, Capussotti L, Barroso E, Nuzzo G, Laurent C, Ijzermans JN, Gigot JF, Figueras J, Gruenberger T, Mirza DF, et al: Progression while receiving preoperative chemotherapy should not be an absolute contraindication to liver resection for colorectal metastases. Ann Surg Oncol 19: 2786-2796, 2012

21. Eisenhauer EA, Therasse P, Bogaerts J, Schwartz LH, Sargent D, Ford R, Dancey J, Arbuck S, Gwyther S, Mooney M, et al: New response evaluation criteria in solid tumors: Revised RECIST guideline (version 1.1). Eur J Cancer 45: 228-247, 2009.

22. Zendel A, Lahat E, Dreznik Y, Zakai BB, Eshkenazy R and Ariche A: 'Vanishing liver metastases'-A real challenge for liver surgeons. Hepatobiliary Surg Nutr 3: 295-302, 2014.

23. Jones RP, Stattner S, Sutton P, Dunne DF, McWhirter D, Fenwick SW, Malik HZ and Poston GJ: Controversies in the oncosurgical management of liver limited stage IV colorectal cancer. Surg Oncol 23: 53-60, 2014.

24. Clavien PA, Petrowsky H, DeOliveira ML and Graf R: Strategies for safer liver surgery and partial liver transplantation. N Engl J Med 356: 1545-1559, 2007.

25. Ruers T, Punt C, Van Coevorden F, Pierie JP, Borel-Rinkes I, Ledermann JA, Poston G, Bechstein W, Lentz MA, Mauer M, et al: Radiofrequency ablation combined with systemic treatment versus systemic treatment alone in patients with non-resectable colorectal liver metastases: A randomized EORTC Intergroup phase II study (EORTC 40004). Ann Oncol 23: 2619-2626, 2012.

26. Tanis E, Nordlinger B, Mauer M, Sorbye H, van Coevorden F, Gruenberger T, Schlag PM, Punt CJ, Ledermann J and Ruers TJ: Local recurrence rates after radiofrequency ablation or resection of colorectal liver metastases. Analysis of the European Organisation for Research and Treatment of Cancer \#40004 and \#40983. Eur J Cancer 50: 912-919, 2014.

27. Adam R, Pascal G, Castaing D, Azoulay D, Delvart V, Paule B, Levi $\mathrm{F}$ and Bismuth $\mathrm{H}$ : Tumor progression while on chemotherapy: A contraindication to liver resection for multiple colorectal metastases? Ann Surg 240: 1052-1064, 2004.

28. Neumann UP, Thelen A, Röcken C, Seehofer D, Bahra M, Riess H, Jonas S, Schmeding M, Pratschke J, Bova R and Neuhaus P: Nonresponse to pre-operative chemotherapy does not preclude long-term survival after liver resection in patients with colorectal liver metastases. Surgery 146: 52-59, 2009.

29. Gallagher DJ, Zheng J, Capanu M, Haviland D, Paty P, Dematteo RP, D'Angelica M, Fong Y, Jarnagin WR, Allen PJ and Kemeny N: Response to neoadjuvant chemotherapy does not predict overall survival for patients with synchronous colorectal hepatic metastases. Ann Surg Oncol 16: 1844-1851, 2009

30. Adam R, De Gramont A, Figueras J, Guthrie A, Kokudo N, Kunstlinger F, Loyer E, Poston G, Rougier P, Rubbia-Brandt L, et al: The oncosurgery approach to managing liver metastases from colorectal cancer: A multidisciplinary international consensus. Oncologist 17: 1225-1239, 2012.

31. Ye LC, Liu TS, Ren L, Wei Y, Zhu DX, Zai SY, Ye QH, Yu Y, $\mathrm{Xu} \mathrm{B}$, Qin XY and Xu J: Randomized controlled trial of cetuximab plus chemotherapy for patients with KRAS wild-type unresectable colorectal liver-limited metastases. J Clin Oncol 31: 1931-1938, 2013.

32. Levi FA, Boige V, Hebbar M, Smith D, Lepère C, Focan C, Karaboué A, Guimbaud R, Carvalho C, Tumolo S, et al: Conversion to resection of liver metastases from colorectal cancer with hepatic artery infusion of combined chemotherapy and systemic cetuximab in multicenter trial OPTILIV. Ann Oncol 27: 267-274, 2016.

33. De Roock W, Piessevaux H, De Schutter J, Janssens M, De Hertogh G, Personeni N, Biesmans B, Van Laethem JL, Peeters M, Humblet Y, et al: KRAS wild-type state predicts survival and is associated to early radiological response in metastatic colorectal cancer treated with cetuximab. Ann Oncol 19: 508-515, 2008

34. Roth AD, Tejpar S, Delorenzi M, Yan P, Fiocca R, Klingbiel D, Dietrich D, Biesmans B, Bodoky G, Barone C, et al: Prognostic role of KRAS and BRAF in stage II and III resected colon cancer: Results of the translational study on the PETACC-3, EORTC 40993, SAKK 60-00 trial. J Clin Oncol 28: 466-474, 2010.

35. Van Cutsem E, Köhne CH, Láng I, Folprecht G, Nowacki MP, Cascinu S, Shchepotin I, Maurel J, Cunningham D, Tejpar S, et al: Cetuximab plus irinotecan, fluorouracil, and leucovorin as first-line treatment for metastatic colorectal cancer: Updated analysis of overall survival according to tumor KRAS and BRAF mutation status. J Clin Oncol 29: 2011-2019, 2011. 